\title{
Epithelial-endothelial cross-talk hypothesis explains the early pathobiology of COVID-19 pneumonia
}

\author{
Amit Jain ${ }^{1}$ \\ ${ }^{1}$ Affiliation not available
}

June 7, 2021

\section{Author Information:}

Amit Jain M.D., Anesthesiology Institute, Cleveland Clinic Abu Dhabi, United Arab Emirates

\section{Corresponding Author:}

Amit Jain

Anesthesiology Institute, Cleveland Clinic Abu Dhabi, Al Maryah Island, Abu Dhabi

United Arab Emirates

M: +971544103790

Email:amitvasujain@gmail.com

Prior Presentations: None

\section{Word and Element Counts:}

Word Count: 2099

Number of references: 40

Funding Statement: Support was provided solely from institutional and/or departmental sources

Conflicts of Interest: The authors declare no competing interests

\section{Abstract}

Since the outbreak of the novel coronavirus disease 2019 (COVID-19), researchers around the globe are constantly puzzled by a well reported clinical finding of pronounced arterial hypoxemia, yet without proportional signs of respiratory distress (i.e. silent hypoxemia or happy hypoxemia) in this disease. Based upon the findings of increased intrapulmonary shunt in COVID-19, many proceed to propose the concept of pulmonary vasoplegia, and not just the loss of hypoxic pulmonary vasoconstriction, as mechanism behind this phenomenon of silent hypoxemia. Further, assumptions were made in proposing inflammatory vasodilators such as prostaglandins and bradykinins to be the pathological mediators. However, a closer look in to the physiology of renin-angiotensin system may suggest the predominant role of angiotensin-converting enzyme and angiotensin II-mediated pulmonary vasoconstriction to be an early mechanism for silent hypoxia. This theory not only supports other clinical symptomatology of COVID-19, including lack of nasal symptoms and absence of asthma as a risk-factor, but also corelates with the histopathological data and the radiological findings of COVID-19 disease.

\section{Keywords}


COVID-19, silent hypoxemia, renin-angiotensin system, SARS-CoV-2, bradykinin

For some time, researchers have struggled to understand the pathophysiology of "silent hypoxia" in coronavirus diseases (COVID-19) patients [1-3]. Based on the high ratio of $3.0 \pm 2.1$ of the shunt fractions to the fractions of gasless tissue as derived from the computed tomography scan findings in COVID-19 patients, Gattinoni et al [1]. were first to suggest pulmonary vasoplegia-induced hyperperfusion of non-aerated lung regions as the responsible pathophysiology. However, they did not offer a specific mechanism for this vasoplegia. Recently, Reynolds et al., using contrast-enhanced transcranial doppler (TCD), demonstrated a right-to-left shunt in COVID-19 patients [2]. As the severity of hypoxemia inversely correlated with the number of microbubbles detected and the prevalence of transpulmonary bubbles seen in this study was markedly higher than the prevalence of patent foramen ovale in the general population, Reynolds et al. concluded that pathological pulmonary vascular dilatation and not just a lack of hypoxic pulmonary vasoconstriction was a significant contributor to hypoxemia in COVID-19 patients with respiratory failure. However, once again no attempt was made to propose an explanation for this pathophysiological process, although the authors compared such the intense pulmonary vascular dilatation with that observed in hepatopulmonary syndrome [2]. Brito-Azevedo et al. also proposed intrapulmonary shunt, as evident using transthoracic saline contrast echocardiography (TTSCE), as an explanation for severe hypoxia despite preserved lung compliance in COVID-19 pneumonia [3]. They suggested a mechanism for the intrapulmonary shunt: SARS-Cov-2 infection-induced angiotensin II (Ang II)-mediated angiotensin converting enzyme (ACE) downregulation with upregulation of bradykinin-induced pulmonary vasodilation. This may correspond with the 'bradykinin storm' hypothesis that has been proposed by other researchers to explain the pulmonary manifestations of COVID-19 [4].

We disagree with these conclusions, however, for the following reasons: First, the bradykinin storm hypothesis could explain some of the pulmonary manifestations but the same pathological model does not explain other extrapulmonary effects of COVID-19, such as myocardial, renal and central nervous system disease manifestations. In fact, most translational medicine research suggests organo-protective effects of bradykinin especially via $B_{2}$ receptor [5]. Second, if bradykinin is assumed to be the key inflammatory mediator, bradykinin-mediated nasal congestion, sneezing, rhinorrhoea and sinusitis as seen in common viral rhinitis [6], should be common symptoms of COVID-19 diseases and pulmonary manifestations like wheezing and bronchospasm should be profound. On the contrary, only $4 \%$ of COVID-19 patients complain of nasal symptoms other than anosmia [7], and asthma has not been identified as a mortality risk factor for severe COVID-19 respiratory disease [8]. Instead, chronic non-asthmatic pulmonary diseases are associated with poor COVID-19 outcomes [8].

\section{Statement of hypothesis}

Though in several observational data, a significant proportion of COVID-19 patients have presented with silent hypoxia with preserved respiratory compliance, thus giving it a terminology of atypical ARDS [1,9], only a few have attempted to relate this phenomenon with increased intrapulmonary shunt $[1,2,3]$. Despite this, based on these observations, many researchers proceed to evoke a hypothesis of bradykinin as a mediator for pulmonary vasoplegia and inflammation in COVID-19. However, no study till date have demonstrated elevated bradykinin levels in either serum or bronchoalveolar lavage fluid in COVID-19 patients. Although, we agree with the Gattinoni et al. [1] Reynolds et al. [2] and Brito-Azevedo et al. [3] findings of increased intrapulmonary shunt in COVID-19 pneumonia but, we are doubtful of their conclusion of pulmonary vasodilation as the predominant phenomenon resulting in increased intrapulmonary shunt and severe hypoxia. Instead, to explain the phenomenon of silent hypoxemia in COVID-19 respiratory failure, we developed an alternate pathophysiological model - Epithelial-Endothelial crosstalk hypothesis - involving upregulation of ACE-Ang II- AT1R pathways producing a mosaic pulmonary perfusion pattern of non-homogenous pulmonary vasoconstriction with resultant intrapulmonary shunting and dead-space [10,11]. We base this on the following arguments:

First , blood flow through intrapulmonary arteriovenous anastomoses has been demonstrated in approximately $30 \%$ of healthy adult humans at rest, and in $100 \%$ of healthy adult humans during exercise and inhalation 
of reduced oxygen gas mixtures [12]. Similarly, pathological conditions such as high-altitude pulmonary edema, bronchopulmonary dysplasia and congenital diaphragmatic hernia are associated with intrapulmonary shunts as the predominant mechanism for hypoxia [12]. In all these conditions raised pulmonary vascular resistance (PVR) is a common denominator and non-homogenous pulmonary vasoconstriction has been linked as the mechanism for the recruitment of intrapulmonary arteriovenous anastomosis and increased shunt [13], but not pulmonary vasodilation. Catecholamine infusions at rest, by increasing cardiac output, can increase the shunt fraction by increasing blood flow through these pathways and negatively impact pulmonary gas exchange [12]. Notably, $66 \%$ patients in the Brito-Azevedo et al. [3] study were on vasopressors while Reynolds et al. [2] did not provide information about vasopressors in their study. Furthermore, platypnea and orthodeoxia, the classical clinical features of significant pulmonary vasodilation-induced shunts, as seen in hepatopulmonary syndrome [14] (an entity closely compared to the intrapulmonary shunt of COVID-19 pneumonia [2]), are not a part of COVID-19 symptomatology.

Second, although TCD and TTSCE are well-known modalities to determine intrapulmonary shunt, neither TCD nor TTSCE can determine the pathophysiological process responsible of the development of shunt: anatomical, vasodilation or non-homogenous pulmonary vasoconstriction.

Third , since COVID-19 lung compliance at the stage of silent hypoxia is largely preserved and the areas of consolidation are minimal [1,3], the fraction of gasless tissue would be expected to be minimal [1]. Thus, impaired hypoxic pulmonary vasoconstriction (HPV) as a cause for severe hypoxia in early COVID-19 patients should not be the sole mechanism. Studies based on in-silico [15] and mathematical [16] modelling also reinforce that even complete loss of HPV could not recreate severe hypoxia observed in COVID-19 pneumonia.

Fourth, the hypothesis of Ang II-induced downregulation of ACE activity is not supported by translational research on renin-angiotensinogen-angiotensin pathways. While increases in bradykinin levels are possible secondary to angiotensin-type-2 receptor $\left(\mathrm{AT}_{2} \mathrm{R}\right)$-mediated Ang II action, this is often a counterregulatory mechanism [17] and hence, should not predominate. Previous studies on ARDS found a correlation between pulmonary capillary endothelium bound ACE activity and severity of ARDS [18]. High ACE levels in bronchoalveolar lavage (BAL) fluids have been observed in ARDS despite a reduction in serum ACE levels [19]. Though, no study on COVID-19 has actually measured the ACE levels in BAL fluid and ACE protein expression in lungs, a recent study measuring the circulating ACE levels in COVID-19 patients demonstrated decreased circulatory levels of ACE in severe COVID-19 patients that normalizes during the recovery phase [20]. This reduced serum ACE levels may reflect loss of enzyme release from a damaged pulmonary vascular endothelium, but not be the true representative of ACE activity in the lung compartment [20] that may actually be increased [21]. High tissue ACE activity increases degradation of bradykinin to inactive metabolites, inhibiting vasodilator pathways in lungs. Similar mechanism can explain the low incidence of rhinorrhea and nasal congestion in SARS-CoV-2, unlike other viral upper respiratory tract infections wherein bradykinin is considered to be the most important mediator. The presence of ACE in the superficial lamina propria and the endothelium of superficial blood vessels of human nasal mucosa also suggests the possible role of ACE in limiting the inflammatory effects of bradykinin in nasal mucosa [22]. A report of increased cerebrospinal fluid levels of ACE in two patients with transient COVID-19 encephalitis (i 3 days) further highlights the pathological role of local ACE upregulation in COVID-19 [23].

Fifth, and most important is to understand the normal physiological and pathological roles of Ang II in humans. In health, $\mathrm{AT}_{2} \mathrm{R}$ and masR, but not angiotensin-type- 1 receptor $\left(\mathrm{AT}_{1} \mathrm{R}\right)$, are the only angiotensin receptor types expressed on pulmonary endothelium and AT1R are expressed on the pulmonary vascular smooth cells and mesenchymal cells located beneath the pulmonary endothelium [24]. Also, the locally produced Ang II in the vascular lumen could not crosses the intact endothelium. Thus, in healthy alveolar capillaries, AngII-AT 2 R and Ang(1-7)-masR induced nitric-oxide (NO)-mediated vasodilatory effect is overactive and maintains low PVR, explaining why Ang II infusions in physiological dose ranges failed to increase PVR $[25,26]$. However, with high-dose Ang II infusions $\mathrm{AT}_{1} \mathrm{R}$ activity predominates and can produce intense non-homogenous pulmonary vasoconstriction and ventilation-perfusion mismatching akin to 
that produced by hypoxia [27, 28]. Ang II infusion has also been demonstrated to produce non-cardiogenic acute pulmonary edema [21]. Also, on one hand, the subpressor levels of Ang II activate AMP-activated protein kinase (AMPK) and increases ACE2, the high levels of Ang II (pathological state) inhibit AMPK, upregulates ACE and downregulates ACE2 via the $\mathrm{AT}_{1} \mathrm{R}-\mathrm{ERK} / \mathrm{p} 38$ MAPK pathway [29]. Thus, Ang II has beneficial as well as harmful vascular effects depending upon the Ang II levels as well as effector receptors expressions. Importantly, COVID-19 patients have high systemic Ang II levels that are linked with disease severity [30] and decrease in Ang II levels following human recombinant soluble ACE2 therapy correlated with the clinical improvement [31].

\section{A working-hypothesis}

The key components of our "Epithelial-Endothelial cross-talk" hypothesis for increased intrapulmonary shunt and silent hypoxemia in COVID-19 are as follows (Fig. 1.):

1. Severe acute respiratory syndrome coronavirus-2 (SARS-CoV-2) enters type-2 pneumocytes following binding to membrane angiotensin converting enzyme 2 (ACE2), causing downregulation of ACE2 on the alveolar-capillary membrane along with concurrent activation of disintegrin and metalloproteinase domain-containing protein 17 (ADAM17) also known as tumor necrosis factor- $\alpha-(\mathrm{TNF}-\alpha)$-converting enzyme (TACE), and resulting in TNF- $\alpha$ and IL-6 amplification. This, in turn, establishes a positive feedback loop of increased expression of ACE, Ang II and $\mathrm{AT}_{1} \mathrm{R}$ on alveolar-capillary membrane and pulmonary capillary smooth muscle cells. Ang II-ATIR-mediated activation of alveolar endothelial cells now increases the release of endothelin-1 and reactive oxygen species (ROS). Meanwhile, ACE 1-Ang II-AT2-R and ACE2-Ang 1-7-masR-mediated constitutive endothelial nitric oxide synthase (eNOS) activation and nitric oxide (NO) release is inhibited.

2. High local Ang II, endothelin-1 and ROS results in intense but heterogenous pulmonary vasoconstriction of the precapillary arterioles, resulting in reduced perfusion of alveolocapillary units. Several other factors that may be contributory to pulmonary vasoconstriction includes TNF- $\alpha$ that works synergistically with Ang II and can induce mitochondrial ROS and can deplete endothelial cells of NO. Plasminogen activation inhibitor-1 (PAI-1) and platelet activating factors (PAF) are some other mediators that can not only potentiate vasoconstriction, but microvascular thrombosis as well. As the resulting vasoconstriction is severe but, uneven, the capillary beds with relatively less vasoconstriction are disproportionately exposed to elevated microvascular pressures, resulting in recruitment and regional over-perfusion. This results in increased shunt fraction and hypoxia, capillary-stress failure, and ground-glass opacities. The proposed interplay of key mediators of pulmonary vasoconstriction involved in the early COVID-19 pathophysiology is depicted in Fig. 2.

3. As the disease worsens, endothelial dysfunction results in proinflammatory and procoagulant activity. Thrombotic occlusion of heterogeneous regions of pulmonary vasculature distal to subsegmental vessels including alveolar capillary microthrombi can further increase the shunt fraction and dead space. Recent evidence of high dead-space ventilation in COVID-19 patients supports our hypothesis [32]. This also explains the findings of dilated proximal sub-segmental and segmental vessels on dual-energy CT [10]. Thus, silent hypoxia of COVID-19 can be mainly secondary to diffuse microvascular lung injury with limited alveolar epithelial injury not amounting to diffuse alveolar damage of ARDS. 


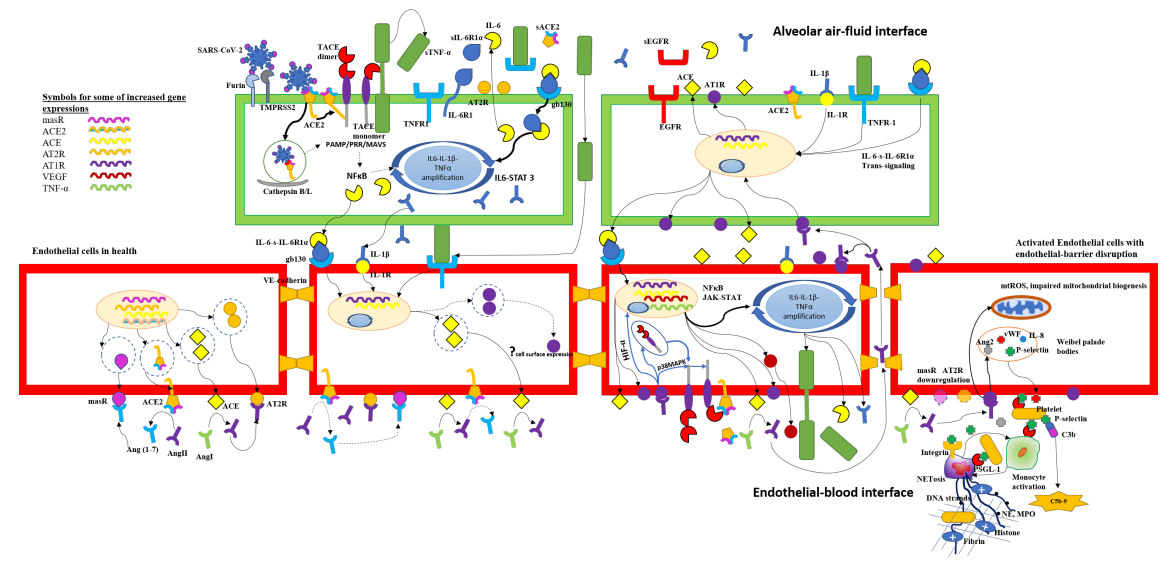

Figure 1: This is a caption

Fig. 1. Simple illustration of Epithelial-endothelial cross-talk hypothesis in COVID-19:

SARS-CoV-2 uses ACE2 as cell entry receptors and causes downregulation of ACE2. This is followed by host pattern recognition receptors (PRRs)-mediated detection of a viral pathogen-associated molecular patterns (PAMPs) resulting in interaction of PRRs with mitochondrial antiviral-signaling protein (MAVS) that activates $\mathrm{NF}_{\chi} \mathrm{B}$ through a signaling cascade involving several kinases. Activated $\mathrm{NF}_{\chi} \mathrm{B}$ translocate to the nucleus and induces the transcription of pro-inflammatory cytokines: Il-6, TNF- $\alpha$ and IL-1 $\beta$. (minor direct pathway ). Binding of SARS-CoV-2 to ACE2 is also associated with activation of TACE dimer to TACE monomer. Activated TACE induces shedding of several membrane proteins including TNF- $\alpha$, IL-6R $\alpha$, TNF$\alpha \mathrm{R} 1, \mathrm{TNF}-\alpha \mathrm{R} 2$ and EGF receptor. TNF- $\alpha$ upregulates AT1R density on epithelial and endothelial cells. Cross-talk between TNF- $\alpha$ and Ang II contribute to mitochondrial oxidative stress, activation of PAI-1, MCP-1 and AP-1. EGF receptor activation induces hypoxia inducible factor 1 alpha (HIF-1 $\alpha$ ) activity. IL6-sILR $\alpha$ complex produces gp130 mediated activation of STAT3 in a variety of 1L6R $\alpha$ negative cells including pericytes, endothelial cells and epithelial cells resulting in activation of $\mathrm{NF}_{\varkappa} \mathrm{B}$ pathway. $\mathrm{NF}_{\chi} \mathrm{B}$ via. downstream mediators Elk-1 and activator protein (AP-1) results in AT1R upregulation. AngII-AT1R overactivity induces a feed-forward loop of TACE activation, sustained EGFR activation and stablishes positive feedback loop of AngII-AT1R-NF ${ }_{\chi}$ B axis. AngII via hypoxia inducible factor 1 alpha (HIF-1 $\alpha$ ) also increases gene expression of ACE, AT1R and VEGF. Overall, positive feedforward pathways are established resulting in upregulation of ACE-AngII-AT1R, TNF $\alpha$ and IL6 activity mediating apoptosis and activation of pericytes, endothelial and epithelial cells and cytokine storm (major indirect pathway ). AngII mediated cytoplasmic and mitochondrial oxidative stress results in impaired mitochondrial biogenesis. AngII-AT1R activity on endothelial cells causes degranulation of weibel palade bodies with massive release of P-selectin, wVF, IL-8 and Ang2, mediators that can generate vascular phenomenon of pericyte loss, platelet aggregation and activation, complement activation, neutrophilic infiltration and NETosis resulting in macro and microvascular thrombosis of COVID-19. 

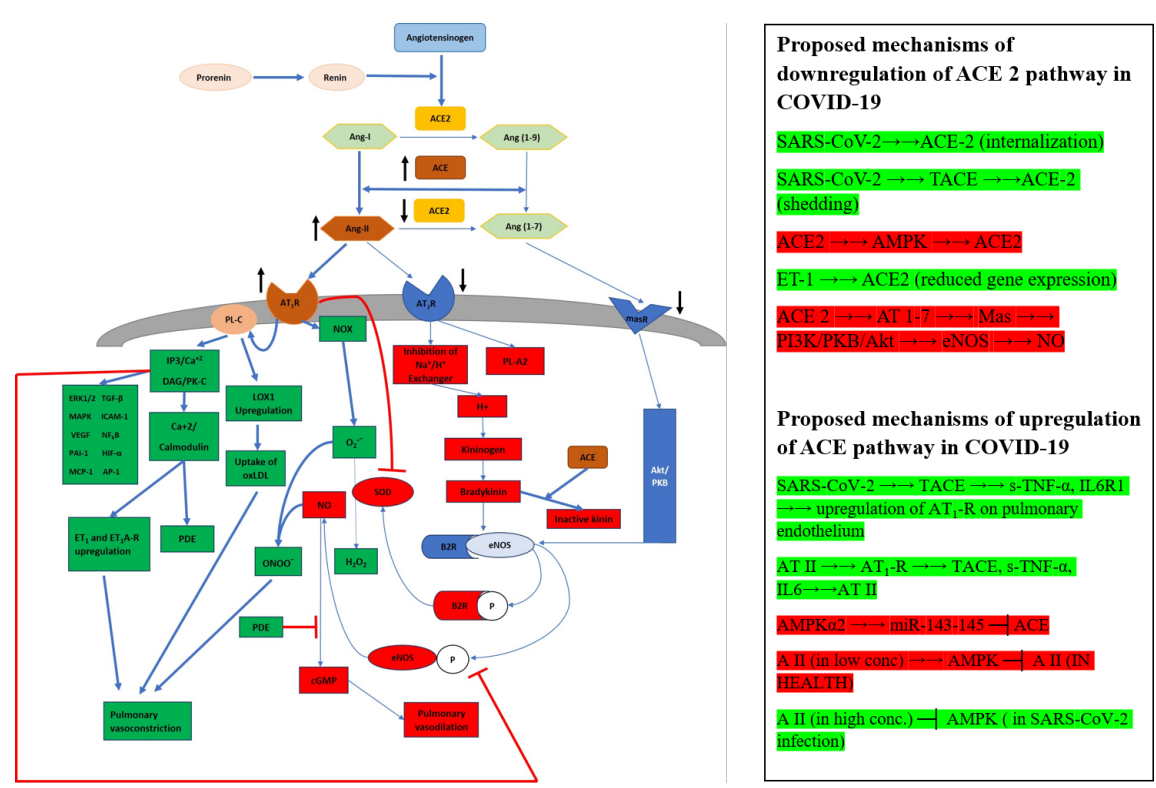

Figure 2: This is a caption

Fig.2. Mechanism for pulmonary vasoconstriction in COVID-19 pneumonia.

Pathways highlighted green are relatively activated while red highlights represent relative inactivation.

ACE: angiotensin-converting enzyme; ACE 2: angiotensin-converting enzyme 2; AT II:

Angiotensin II; AT I: Angiotensin I; AT1R: Ang II type-1 receptor; AT2R: Ang II type-2

receptor; NOX: nicotinamide adenine dinucleotide phosphate oxidase; LOX-1: Lecithin-

Like oxLDL Receptor-1; ETAR: Endothelin-1A receptor; B2R: B2 receptor; eNOS:

endothelial nitric oxide synthase; SOD: superoxide dismutase; NO: nitric oxide; PLC:

phospholipase C; PKC: protein kinase C; TACE: Tumor necrosis factor alpha converting

enzyme; s-TNF- $\alpha$ : soluble tumor necrosis factor alpha; IL6R1: interleukin 6 receptor;

IL6: interleukin 6; AT 1-7: angiotensin 1-7; PI3K/PKB/Akt: phosphoinositide-3-

kinase-protein kinase B/Akt; AMPK: adenosine monophosphate-activated protein

kinase; miR: microRNA; EC: endothelial cell; VSMC: vascular smooth muscle cell.

In conclusion, Ang II-mediated heterogenous pulmonary vasoconstriction with microthrombotic perfusion defects but not pulmonary vasodilation is likely the predominant mechanism for silent hypoxemia in early COVID-19 pneumonia. Our hypothesis is supported by the fact that the patients with precapillary pulmonary hypertension and hypoxia often demonstrate significant intrapulmonary arteriovenous shunt with increase in alveolar-arterial $\mathrm{PaO} 2$ gradient [33]. This hypothesis is further supported by the high incidence of raised PVR and right ventricular dysfunction in early COVID-19 disease [34] and reports of lung perfusion deficits that do not overlap with ground-glass opacities or consolidation and are not associated with major vessel occlusion $[10,35]$. Based on this model, we have previously advocated the beneficial role of steroids and anticoagulant in the early to late stages of COVID-19 [10] and this recommendation is now supported by more clinical evidence $[36,37]$. We strongly believe determining the predominant pathophysiology - heterogenous pulmonary vasoconstriction versus pulmonary vasodilation - has an important treatment implication: early pulmonary vasoconstrictors versus vasodilators. Disappointingly, no study has yet evaluated the role of NO 
therapy at an early stage of COVID-19 disease and our recommendation on the use of early NO therapy in the treatment of symptomatic high risk COVID-19 patients [10] is still under debate [38, 39], the issue certainly warrants high quality randomized clinical trials. Nonetheless, a recent multicentric retrospective study comparing the outcome of COVID-19 disease in the elderly population on antihypertensive with those who were not on any antihypertensive therapy, authors identified significant benefits of ACE inhibitors (ACEIs), AT1R blockers (ARBs) as well as calcium channel blockers in reducing the severity and mortality of COVID-19 [40]. Our hypothesis of "epithelial-endothelial cross-talk" further raises interest in the possible therapeutic role of ACEIs or ARBs in COVID-19.

\section{Conflict of interest statement: None}

\section{References}

1. Gattinoni L, Coppola S, Cressoni M, Busana M, Rossi S, Chiumello D. Covid-19 Does Not Lead to a "Typical" Acute Respiratory Distress Syndrome. Am J Respir Crit Care Med, 201(10)(2020), pp. 1299-1300, 10.1164/rccm.202003-0817LE

2. Reynolds AS, Lee AG, Renz J, DeSantis K, Liang J, Powell CA, et al. Pulmonary Vascular Dilatation Detected by Automated Transcranial Doppler in COVID-19 Pneumonia. Am J Respir Crit Care Med, 202(7)(2020), pp. 1037-1039, 10.1164/rccm.202006-2219LE

3. Brito-Azevedo A, Pinto EC, Corrêa GAdCP, Bouskela E. SARS-CoV-2 infection causes pulmonary shunt by vasodilatation. J Med Virol, (2020), 10.1002/jmv.26342

4. Garvin MR, Alvarez C, Miller JI, Prates ET, Walker AM, Amos BK, et al. A mechanistic model and therapeutic interventions for COVID-19 involving a RAS-mediated bradykinin storm. Elife 2020; 9: e59177. Published 2020 Jul 7. doi:10.7554/eLife.59177

5. Golias Ch, Charalabopoulos A, Stagikas D, Charalabopoulos K, Batistatou A. The kinin systembradykinin: biological effects and clinical implications. Multiple role of the kinin system-bradykinin. Hippokratia, 11(3)(2007), pp. 124-128.

6. Eccles R. Understanding the symptoms of the common cold and influenza. Lancet Infect Dis, 5(11)(2005), pp. 718-725, doi: 10.1016/S1473-3099(05)70270-X

7. Wang Y, Chen J, Chen W, Liu L, Dong M, Ji J, Hu D, Zhang N. Does Asthma Increase the Mortality of Patients with COVID-19?: A Systematic Review and Meta-Analysis. Int Arch Allergy Immunol, 182(1)(2021), pp. 76-82, doi: 10.1159/000510953

8. El-Anwar MW, Elzayat S, Fouad YA. ENT manifestation in COVID-19 patients. Auris Nasus Larynx, 47(4)(2020), pp. 559-564, doi: 10.1016/j.anl.2020.06.003

9. Chiumello, D., Busana, M., Coppola, S. et al. Physiological and quantitative CT-scan characterization of COVID-19 and typical ARDS: a matched cohort study. Intensive Care Med, 46(2020), pp. 2187-2196, https://doi.org/10.1007/s00134-020-06281-2

10. Jain A, Doyle DJ. Stages or phenotypes? A critical look at COVID-19 pathophysiology. Intensive Care Med, 46(7)(2020), pp. 1494-1495, doi: 10.1007/s00134-020-06083-6

11. Jain A, Doyle DJ, Mangal R. "Mosaic Perfusion Pattern" on Dual-Energy CT in COVID-19 Pneumonia: Pulmonary Vasoplegia or Vasoconstriction?. Radiol Cardiothorac Imaging, 2(5)(2020), e200433, doi:10.1148/ryct.2020200433

12. Lovering AT, Riemer RK, Thébaud B. Intrapulmonary arteriovenous anastomoses. Physiological, pathophysiological, or both?. Ann Am Thorac Soc, 10(5)(2013), pp. 504-508, doi:10.1513/AnnalsATS.201308-265ED

13. Dehnert C, Risse F, Ley S, Kuder TA, Buhmann R, Puderbach M, et al. Magnetic resonance imaging of uneven pulmonary perfusion in hypoxia in humans. Am J Respir Crit Care Med, 174(10)(2006), pp. 1132-1138, doi: 10.1164/rccm.200606-780OC

14. Krowka MJ, Dickson ER, Cortese DA. Hepatopulmonary syndrome. Clinical observations and lack of therapeutic response to somatostatin analogue. Chest, 104(4)(1993), pp. 515-521, doi: 10.1378/chest.104.2.515

15. Das A, Saffaran S, Chikhani M, Scott TE, Laviola M, Yehya N, et al. In Silico Modeling of Coronavirus Disease 2019 Acute Respiratory Distress Syndrome: Pathophysiologic Insights 
and Potential Management Implications. Critical Care Explorations, 2(9)(2020), p. e0202, https://doi.org/10.1097/CCE.000000000000202

16. Herrmann J, Mori V, Bates JHT, Suki B. Modeling lung perfusion abnormalities to explain early COVID-19 hypoxemia. Nat Commun, 11(2020), p. 4883, https://doi.org/10.1038/s41467-020-18672-6

17. Searles CD, Harrison DG. The interaction of nitric oxide, bradykinin, and the angiotensin II type 2 receptor: lessons learned from transgenic mice. J Clin Invest, 104(8)(1999), pp. 1013-1014.

18. Orfanos SE, Armaganidis A, Glynos C, Psevdi E, Kaltsas P, Sarafidou P, Catravas JD, Dafni UG, Langleben D, Roussos C. Pulmonary capillary endothelium-bound angiotensin-converting enzyme activity in acute lung injury. Circulation, 102(16)(2000), pp. 2011-2018, doi: 10.1161/01.cir.102.16.2011

19. Idell S, Kueppers F, Lippmann M, Rosen H, Niederman M, Fein A. Angiotensin converting enzyme in bronchoalveolar lavage in ARDS. Chest, 91(1)(1987), pp. 52-56, doi: 10.1378/chest.91.1.52

20. Zhu, Z., Cai, T., Fan, L. et al. The potential role of serum angiotensin-converting enzyme in coronavirus disease 2019. BMC Infect Dis, 20(2020), p. 883, https://doi.org/10.1186/s12879-020-05619-x

21. Yilin Z, Yandong N, Faguang J. Role of angiotensin-converting enzyme (ACE) and ACE2 in a rat model of smoke inhalation induced acute respiratory distress syndrome. Burns, 41(7)(2015). pp. 146877, doi.org/10.1016/j.burns.2015.04.010.

22. Ohkubo K, Lee CH, Baraniuk JN, Merida M, Hausfeld JN, Kaliner MA. Angiotensin-converting enzyme in the human nasal mucosa. Am J Respir Cell Mol Biol, 11(2)(1994), pp. 173-80, 10.1165/ajrcmb.11.2.8049077

23. Bodro M, Compta Y, Llansó L, Esteller D, Doncel-Moriano A, Mesa A, et al. Increased CSF levels of IL-1 $\beta$, IL-6, and ACE in SARS-CoV-2-associated encephalitis. Neurol Neuroimmunol Neuroinflamm, 7(2020), e821. doi:10.1212/NXI.0000000000000821

24. Bullock GR, Steyaert I, Bilbe G, Carey RM, Kips J, De Paepe B, et al. Distribution of type-1 and type2 angiotensin receptors in the normal human lung and in lungs from patients with chronic obstructive pulmonary disease. Histochem Cell Biol 2001; 115: 1171124. https://doi.org/10.1007/s004180000235

25. Mandel MJ, Sapirstein LA. Effect of angiotensin infusion on regional blood flow and regional vascular resistance in the rat. Circ Res, 10(1962), pp. 807-16, doi: 10.1161/01.res.10.5.807

26. Iwamoto HS, Rudolph AM. Effects of angiotensin II on the blood flow and its distribution in fetal lambs. Circ Res, 48(2)(1981), pp. 183-189, doi: 10.1161/01.res.48.2.183

27. Zhao X, Li X, Trusa S, Olson SC. Angiotensin type 1 receptor is linked to inhibition of nitric oxide production in pulmonary endothelial cells. Regul Pept 2005; 132: 113-122.

28. Hansen TN, Le Blanc AL, Gest AL. Hypoxia and angiotensin II infusion redistribute lung blood flow in lambs. J Appl Physiol, 58(3)(1985), pp. 812-818, https://doi.org/10.1152/jappl.1985.58.3.812

29. Liu J, Li X, Lu Q, Ren D, Sun X, Rousselle T, et al. AMPK: a balancer of the renin-angiotensin system. Biosci Rep 2019; 39: BSR20181994. Published 2019 Sep 3. doi:10.1042/BSR20181994

30. Liu Y, Yang Y, Zhang C, Huang F, Wang F, Yuan J, et al. Clinical and biochemical indexes from 2019-nCoV infected patients linked to viral loads and lung injury. Sci China Life Sci, 63(3)(2020), pp. 364-374, doi: 10.1007/s11427-020-1643-8

31. Zoufaly A, Poglitsch M, Aberle JH, Hoepler W, Seitz T, Traugott M, et al. Human recombinant soluble ACE2 in severe COVID-19. Lancet Respir Med, 8(11)(2020), pp. 1154-1158, doi: 10.1016/S22132600(20)30418-5

32. Mauri T, Spinelli E, Scotti E, Colussi G, Basile MC, Crotti S, et al. Potential for lung recruitment and ventilation-perfusion mismatch in patients with the acute respiratory distress syndrome from coronavirus disease. Crit Care Med 2020. https://doi.org/10.1097/CCM.0000000000004386

33. Vodoz JF, Cottin V, Glérant JC, Derumeaux G, Khouatra C, Blanchet AS et al. Right-to-left shunt with hypoxemia in pulmonary hypertension. BMC Cardiovasc Disord, 9(2009), p.15, doi: 10.1186/14712261-9-15

34. Szekely Y, Lichter Y, Taieb P, Bani A, Hochstadt A, Merdler I. The Spectrum of Cardiac Manifestations in Coronavirus Disease 2019 (COVID-19) - A Systematic Echocardiographic Study. Circulation, 142(4)(2020), pp. 342-353, doi: 10.1161/CIRCULATIONAHA.120.047971

35. Santamarina MG, Boisier D, Contreras R, Baque M, Volpacchio M, Beddings I. COVID-19: 
a hypothesis regarding the ventilation-perfusion mismatch. Crit Care, 24(1)(2020), pp. 395-399, doi.org/10.1186/s13054-020-03125-9

36. The WHO Rapid Evidence Appraisal for COVID-19 Therapies (REACT) Working Group. Association Between Administration of Systemic Corticosteroids and Mortality Among Critically Ill Patients With COVID-19: A Meta-analysis. JAMA, 324(13)(2020), pp. 1330-1341, doi: 10.1001/jama.2020.17023

37. Tang N, Bai H, Chen X, Gong J, Li D, Sun Z. Anticoagulant treatment is associated with decreased mortality in severe coronavirus disease 2019 patients with coagulopathy. J Thromb Haemost, 18(5)(2020), pp. 1094-1099, doi: 10.1111/jth.14817

38. Longobardo A, Montanari C, Shulman R, Benhalim S, Singer M, Arulkumaran N. Inhaled nitric oxide minimally improves oxygenation in COVID-19 related acute respiratory distress syndrome. Br $\mathrm{J}$ Anaesth, 126(1)(2021), e44-e46, doi: 10.1016/j.bja.2020.10.011

39. Garfield B, McFadyen C, Briar C, Bleakley C, Vlachou A, Baldwin M, et al. Potential for personalised application of inhaled nitric oxide in COVID-19 pneumonia. Br J Anaesth, 126(2)(2021), e72-e75, doi: 10.1016/j.bja.2020.11.006

40. Yan F, Huang F, Xu J, Yang P, Qin Y, Lv J, et al. Antihypertensive drugs are associated with reduced fatal outcomes and improved clinical characteristics in elderly COVID-19 patients. Cell Discov, 6(2020), p. 77, doi: 10.1038/s41421-020-00221-6 